\title{
Systolic Dyssynchrony Index as a Diagnostic Tool for Subclinical Cardiac Involvement of Sarcoidosis: A cross-sectional single center study.
}

\section{Ali Kemal Cabuk ( $\sim$ kardio.80@hotmail.com )}

Izmir Tepecik Training and Research Hospital: TC Saglik Bakanligi Izmir II Saglik Mudurlugu Izmir Saglik Bilimleri Universitesi Tepecik Egitim ve Arastirma Hastanesi https://orcid.org/0000-0001-5549-3744

\section{Gizem Cabuk}

Izmir Tepecik Training and Research Hospital: TC Saglik Bakanligi Izmir II Saglik Mudurlugu Izmir Saglik Bilimleri Universitesi Tepecik Egitim ve Arastirma Hastanesi https://orcid.org/0000-0002-3478-4611

\section{Research Article}

Keywords: sarcoidosis, cardiac, three-dimensional echocardiography, systolic dyssynchrony index

Posted Date: May 7th, 2021

DOI: https://doi.org/10.21203/rs.3.rs-496795/v1

License: (a) (i) This work is licensed under a Creative Commons Attribution 4.0 International License. Read Full License 


\section{Abstract}

Purpose: Sarcoidosis with cardiac involvement has a relatively high morbidity and mortality, and early diagnose of cardiac sarcoidosis is a critical issue. Systolic dyssynchrony index (SDI) measured by threedimensinonal echocardiography was used in our study for detection of subclinical left ventricular (LV) systolic dysfunction in patients with sarcoidosis and normal LV function on two-dimensional echocardiography.

Methods: Forty-four patients diagnosed with sarcoidosis (without clinically apparent cardiac involvement) and 44 healthy control subjects were included in this study. Conventional 2D echocardiographic parameters and SDI measured by 3D echocardiography were analyzed in all participants.

Results: While two-dimensional echocardiographic results of study groups were similar; SDI_16 (SDI for 16 segments of LV) results were significantly higher in sarcoidosis group compared to healthy controls $(6.99+5.02$ vs $2.89+1.32, p<0.0001)$, and $47.7 \%$ of patients with sarcoidosis had SDI_16 value of $>6 \%$.

Conclusion: SDI_16 was higher in patients with sarcoidosis and this parameter could be used as a marker to identify patients with cardiac involvement of sarcoidosis in the early phase.

\section{Introduction}

Sarcoidosis is an inflammatory disorder and its etiology remains obscure. It is characterized by the formation of non-caseating granulomas and subsequent tissue scarring in the affected organs such as the lymphatic system, gastro-intestinal system, liver, skin, eyes, lungs, and nervous system, as well as in the heart [1].

Prevalence of cardiac sarcoidosis was estimated about $5 \%$, but recent autopsy series and imaging studies demonstrated that it is actually at around $25 \%$ [2]. Disturbance of conduction system like complete heart block and right bundle branch block are the most common findings of cardiac sarcoidosis; however, wall motion abnormalities, anterior basal septal thinning, ventricular dilatation and/or aneurysms, decreased left or right ventricular systolic or diastolic function, ventricular tachycardia, valvular abnormalities, pericardial effusion and sudden cardiac death are other cardiac manifestations of sarcoidosis. Cardiac involvement can be difficult to detect, because of the focal nature of the disease; so, cardiac biopsy has a sensitivity of only $20-30 \%$ [3].

Two-dimensional transthoracic echocardiography is the recommended initial screening test for cardiac sarcoidosis. Wall motion abnormalities in noncoronary distribution or antero-basal septal thinning with increased echogenicity may suggest cardiac involvement of sarcoidosis [4]; but it has relatively low sensitivity and spesifity to detect cardiac involvement at early stage of the disease. Magnetic resonance imaging and nuclear imaging modalities were studied for this purpose and their results are important for clinical diagnosis of cardiac sarcoidosis [5]; nevertheless, this topic is still of a debate in the literature. 
The diagnostic criteria of CS are mainly based on expert consensus and need to be validated by new prospective clinical trials.

Three-dimensional echocardiography (3DE) is an effective imaging tool to measure not only ventricular volumes and ejection fraction but also subclinical systolic dysfunction which could be shown by systolic dyssynchrony index (SDI). SDI is a parameter which can predict worsening systolic function at early phase of the disorder [6]. Because of patchy infiltration nature of sarcoidosis, segmental wall motion abnormalities and dyssynchronic contraction of ventricules may be the initial stage of systolic dysfunction. So, we hypothesized in our study that subclinical cardiac systolic dysfunction in patients with sarcoidosis might be diagnosed with SDI by using 3DE.

\section{Materials And Methods Study Population}

We enrolled 44 patients diagnosed with sarcoidosis, but without clinical apparent cardiac involvement or without suggested criteria for screening for cardiac sarcoidosis [5], and age-sex matched 44 healthy control subjects for echocardiographic analysis. Patients with cardiac symptoms which might be related with cardiac involvement of sarcoidosis (e.g. palpitation, pre-syncope or syncope), known coronary heart disease, systemic or pulmonary hypertension, severe heart valve disease or prosthetic heart valve, left and right bundle branch block, second or third degree atrio-ventricular block, sustained or non-sustained ventricular tachycardia, unexplained pathological Q waves in $\geq 2$ leads, heart failure (ejection fraction < $50 \%$ ) and atrial fibrillation were excluded from the study. Informed consents were obtained from all participants, and this study was approved by our local institutional ethics committee.

\section{Echocardiography Protocol}

We used Philips iE33 with a matrix array transducer (X4.1 transducer; Philips Medical Systems, Bothell, WA, USA) for echocardiograhic analyzes. We performed the modified Simpson's method to measure left ventricular ejection fraction (LVEF), left ventricular end-diastolic volume (LVEDV) and left ventricular endsystolic volume (LVESV) in 2DE as described in guidelines. Optimal gain and compress, sector width and depth were all adjusted at two dimensional echo and we switched to xPlane imaging to ensure the visibility of endocardial borders at orthogonal view. The patients were asked for breath-holding to prevent stitching artifacts and we acquired four beats full volumes from apical four-chamber view. We acquired each subvolume ECG gated with regular four consequent R-wave (excluded premature beats). Elevation and lateral width settings were optimized to reach a frame rate at least $25 \mathrm{fps}$. We used Qlab software (Version 9.0; Philips Medical Systems) to measure LVEF and volumes. All protocols performed by two investigators who were experienced in echocardiographic imaging and blinded each other to detect interobserver variability. We defined end-diastole as the first frame after mitral valve closure, and end-systole as the first frame after aortic valve closure; but we visually corrected the frame in some cases based on the left ventricular size. We adjusted transverse (at the level of papillary muscles) and saggital (from the midline of mitral annulus to apex) planes; then automatic border definition was performed by applying 
five points: anterior, inferior, septal, lateral annulus, and apex. Border definitions were manually modified in some of cases by including papillary muscles and trabeculations as parts of LV cavity (Figs. 1 and 2). We performed sequence analysis at the end of the procedure and checked for border detection frame-byframe. LVEF, LVEDV, LVESV and 16 segments SDI (SDI_16) were obtained at the end of analysis and shown as a report page (Fig. 3).

\section{Statistical Analysis}

SPSS 21.0 (SPSS, Chicago, IL, USA) was used for statistical analysis. The Kolmogorov-Smirnov test was applied to the variables to determine whether or not they are normally distributed. Categorical variables were demonstrated as number and percentage; and continuous variables were demonstrated as mean \pm SD when normally distributed while nonparametric variables were shown as median and the percentiles. Student's t-test was used to compare parameters which were normally distributed. Mann-Whitney $\mathrm{U}$ test was used to compare paramaters which were non-normally distributed. All statistical testing was on the basis of a 2-sided $a=0.05$ significance level.

\section{Results}

In sarcoidosis group $55.25 \%$ of participants were female and in healthy control group it was $52.30 \%$ ( $p=$ 0.09). The baseline characteristics and 2DE measurements of study groups shown in Table 1. Although, deceleration time (DT) of E wave and isovolumetric relaxation time (IVRT) measurements were higher in sarcoidosis group, this difference did not translate to a statistical significance in terms of diastolic dysfunction (Table 1). In 3DE measurements, LVEDV results were similar between two groups; however, LVESV results were mildly higher in patients with sarcoidosis and as a consequence of this, LVEF3D (LVEF values obtained from three dimensional echocardiography) values were lower in sarcoidosis group but this difference did not reach to a statistical significance $(52.62+3.39$ vs $56.32+2.06, p=0.06)$ (Table 2). As our main finding in this study, the SDI_16 results were significantly higher in sarcoidosis group compared to healthy controls $(6.99+5.02$ vs $2.89+1.32, \mathrm{p}<0.0001)$ (Table 2$) .21$ of 44 patients (47.7\%) sarcoidosis had SDI_16 value of $>6 \%$ which may be accepted as a cut-off value based on data comes from literature (data not shown). 
Table 1

Baseline characteristics and two-dimensional echocardiography measurements of study population

\begin{tabular}{|llll|}
\hline Parameter & $\begin{array}{l}\text { Sarcoidosis } \\
(\mathbf{n = 4 4})\end{array}$ & $\begin{array}{l}\text { Control } \\
(\mathbf{n = 4 4})\end{array}$ & P value \\
\hline Age & $53.02( \pm 14.04)$ & $51.75( \pm 11.14)$ & 0.12 \\
\hline BMI $\left(\mathrm{kg} / \mathrm{m}^{2}\right)$ & $25.38( \pm 4.99)$ & $24.04( \pm 1.42)$ & 0.18 \\
\hline IVS thickness $(\mathrm{cm})$ & $0.92( \pm 0.42)$ & $0.94( \pm 0.36)$ & 0.08 \\
\hline e/a & $0.94( \pm 0.35)$ & $0.99( \pm 0.41)$ & 0.61 \\
\hline e/ e'lateral & $6.45( \pm 2.48)$ & $5.63( \pm 2.29)$ & 0.13 \\
\hline DT $(\mathrm{ms})$ & $173.86( \pm 43.26)$ & $149.50( \pm 27.31)$ & $0.03^{*}$ \\
\hline IVRT $(\mathrm{ms})$ & $98.95( \pm 17.17)$ & $89.25( \pm 15.96)$ & $0.01^{*}$ \\
\hline TAPSE $(\mathrm{cm})$ & $2.26( \pm 0.26)$ & $2.35( \pm 0.38)$ & 0.22 \\
\hline LVEF2D $(\%)$ & $57.89( \pm 4.96)$ & $59.50( \pm 3.69)$ & 0.09 \\
\hline Hb $(\mathrm{g} / \mathrm{dL})$ & $12.25( \pm 2.22)$ & $13.02( \pm 2.28)$ & 0.07 \\
\hline WBC $\left(\times 10^{9} / \mathrm{L}\right)$ & $7.42( \pm 2.36)$ & $7.12( \pm 2.78)$ & 0.08 \\
\hline
\end{tabular}

BMl; body mass index, IVS; interventricular septum, DT; deceleration time, IVRT; isovolumetric relaxation time, TAPSE; tricuspit annular plane systolic excursion, LVEF2D; two-dimensional left vetricular ejection fraction.

Table 2

Three-dimensional echocardiography measurements of study groups

\begin{tabular}{|llll|}
\hline Parameter & $\begin{array}{l}\text { Sarcoidosis } \\
(\mathbf{n}=44)\end{array}$ & $\begin{array}{l}\text { Control } \\
(\mathbf{n = 4 4})\end{array}$ & P value \\
\hline LVEDV $(\mathrm{mL})$ & $76.58( \pm 20.77)$ & $75.15( \pm 16.76)$ & 0.73 \\
\hline LVESV $(\mathrm{mL})$ & $38.75( \pm 11.50)$ & $34.82( \pm 7.42)$ & 0.07 \\
\hline LVEF3D $(\%)$ & $53.62( \pm 3.39)$ & $56.32( \pm 2.06)$ & 0.06 \\
\hline SDI_16 & $6.99( \pm 5.02)$ & $2.89( \pm 1.32)$ & $<0.0001^{\star}$ \\
\hline
\end{tabular}

LVEDV; left ventricular end-diastolic volume, LVESV; left ventricular end-systolic volume, LVEF3D; threedimensional left ventricular ejection fraction, SDI_16; sixteen segments systolic dyssynchrony index.

\section{Discussion}


Sarcoidosis itself may have a benign course because of spontaneous remissions; often observed in extracardiac cases such as skin sarcoidosis. However, when cardiac involvement exists, the prognosis becomes unfavourable. Therefore, early diagnosis of cardiac involvement of sarcoidosis is crucial.

Isolated type of CS, described in the literature as a cardiac involvement without systemic manifestations of sarcoidosis [7, 8], is also an important entity. Takaya et al [9] recently reported that this type of sarcoidosis (they called it 'probable CS') has a worse outcome compared with definite CS (sarcoidosis with cardiac involvement). Therefore, the early diagnosis of isolated CS is also an important issue in the clinical setting.

Despite many abnormalities that can be identified by echocardiography, many patients with CS may have a normal result of 2D echocardiography [10]. For instance, Mehta et al [11] found that echocardiographic abnormalities present in only $25 \%$ of the patients who exhibited cardiac magnetic resonance imaging (CMR) or 18F-fluorodeoxyglucose (FDG) positron emission tomographic (PET) evidence of CS; and Freeman et al [12] found that echocardiographic findings could exclude CS in only $32 \%$ of patients with sarcoidosis.

Recent studies have shown that speckle-tracking echocardiography might be used to detect early stage of CS [13]. Chen et al. [14] found that impaired left ventricular longitudinal strain could be an indicator of early granulomatous cardiac infiltration and higher degree of reduction in LV longitudinal strain is associated with increased risk of adverse cardiac events and poor clinical outcomes. Bayat et al. [15] recently reported in their study that $2 \mathrm{D}$ speckle tracking echocardiography could reveal subclinical cardiac involvement of sarcoidosis in patients with normal ejection fraction.

Beyond 2D echocardiography there are valuable tools which are also being recommended in diagnostic criteria of CS (5). One of them is cardiac magnetic resonance (CMR). CMR has a high sensitivity and spesifity in detecting CS, and also gives important prognostic information about patients; thus CMR may guide monitoring and therapy $[16,17]$. We can detect acute inflammation with T2-weighted imaging and fibrosis or scar with late gadolinium enhancement (LGE) imaging. However, CMR is a relatively expensive modality and the findings of LGE on CMR can be present in other cardiac conditions such as myocarditis [18], and the gadolinium-enhanced CMR imaging is contraindicated in patients with advanced renal failure (eGFR $<30 \mathrm{~mL} / \mathrm{min}$ ) due to the risk of nephrogenic systemic fibrosis. PET or in combination with computarized tomography (PET/CT) is also a valuable diagnostic tool for early diagnosis, management, follow-up, and prognostication in patients with CS [19-21]. However, PET/CT is not a widely available modality and more prospective data are warranted to better characterize the role of it in CS.

During the last decade, with advencements of non-invasive diagnostic tests such as 3D echocardiography, lots of cardiac pathologies could be detected quickly and correctly. Measurement of left ventricular volumes and ejection fraction have been more reliable compared to 2DE; and segmental contractility and/or overall synchronicity of ventricular contraction could be defined precisely with 3DE. One of the best parameters of ventricular dyssynchrony is SDI; and it shows deterioration of ventricular contractility in the early stage. SDI has been used in numerous studies to identify affected cardiac 
functions in different clinical scenarios [22-24]. The only clinical trial using 3DE in patients CS that we could find in the literature was the study of Tsuji et al [25]. They (25) found that 3D speckle-tracking radial strain shows good potential to distinguish CS from dilated cardiomyopathy. We enrolled patients diagnosed with sarcoidosis but without any clinical indicator of cardiac involvement as well as with normal ejection fraction; and found that 16-SDI might be a valuable parameter to detect subclinical cardiac involvement of CS. Dyssynchronic contraction could be expected in patients with CS due to the patchy nature of infiltrations seen in sarcoidosis.

The limitations of our study were: This study had a relatively small sample size and we did not perform $\mathrm{PET} / \mathrm{CT}, \mathrm{CMR}$ imaging or endomyoardial biopsy to our study population, because the participants were all without clinical apparent cardiac involvement or without suggested criteria for screening for cardiac sarcoidosis. This was a cross-sectional study so we could not have a prognostic information, but we have been monitoring more closely the patients with SDI_16 result higher than $6 \%$.

\section{Conclusion}

SDI_16 measured by 3DE was significantly higher in patients with sarcoidosis compared to healty control subjects. This might be a promising parameter to identify patients with cardiac involvement of sarcoidosis in the early phase of the disease.

\section{Declarations}

Funding: Not applicable

Conflicts of interest/Competing interests: Not applicable

Availability of data and material: Not applicable

Code availability: Not applicable

\section{References}

1. Iannuzzi MC, Fontana JR (2011) Sarcoidosis: clinical presentation, immunopathogenesis, and therapeutics. JAMA 305: 391-9. doi: 10.1001/jama.2011.10.

2. Martusewicz-Boros MM, Boros PW, Wiatr E, Zych J, Piotrowska-Kownacka D, Roszkowski-Śliż K (2016) Prevalence of cardiac sarcoidosis in white population: a case-control study: Proposal for a novel risk index based on commonly available tests. Medicine 95(32): 4518. doi: 10.1097/MD.0000000000004518.

3. Cooper LT, Baughman KL, Feldman AM, Frustaci A, Jessup M, Kuhl U, Levine GN, Narula J, Starling RC, Towbin J, Virmani R (2007) The role of endomyocardial biopsy in the management of cardiovascular disease: a scientific statement from the American Heart Association, the American College of Cardiology, and the European Society of Cardiology Endorsed by the Heart Failure Society 
of America and the Heart Failure Association of the European Society of Cardiology. Eur Heart J 28: 3076-93. doi: 10.1093/eurheartj/ehm456.

4. Uemura A, Morimoto S, Kato Y, Hiramitsu S, Ohtsuki M, Kato S, Sugiura A, Miyagishima K, Iwase M, Hishida $\mathrm{H}$ (2005) Relationship between basal thinning of the interventricular septum and atrioventricular block in patients with cardiac sarcoidosis. Sarcoidosis Vasc Diffuse Lung Dis 22(1):63-5. doi: 10.1007/s11083-005-9006-9.

5. Birnie DH, Sauer WH, Bogun F, Cooper JM, Culver DA, Duvernoy CS, Judson MA, Kron J, Mehta D, Cosedis Nielsen J, Patel AR, Ohe T, Raatikainen P, Soejima K (2014) HRS expert consensus statement on the diagnosis and management of arrhythmias associated with cardiac sarcoidosis. Heart Rhythm 11: 1305-23. doi: 10.1016/j.hrthm.2014.03.043.

6. Osama I I Soliman, Bas M van Dalen, Attila Nemes, Heleen B van der Zwaan, Wim B Vletter, Folkert J ten Cate, Dominic A M J Theuns, Luc J Jordaens, Marcel L Geleijnse (2009) Quantification of left ventricular systolic dyssynchrony by real-time three-dimensional echocardiography. J Am Soc Echocardiogr 22(3): 232-9. doi: 10.1016/j.echo.2008.11.009.

7. Kandolin R, Lehtonen J, Graner M, et al (2011) Diagnosing isolated cardiac sarcoidosis. J Intern Med 270:461-8. doi: 10.1111/j.1365-2796.2011.02396.x.

8. Isobe M, Tezuka D (2015) Isolated cardiac sarcoidosis: clinical characteristics, diagnosis and treatment. Int J Cardiol 182:132-40. doi: 10.1016/j.jjcard.2014.12.056.

9. Takaya Y, Kusano KF, Nakamura K, et al (2015) Comparison of outcomes in patients with probable versus definite cardiac sarcoidosis. Am J Cardiol 115:1293-7. doi: 10.1016/j.amjcard.2015.01.562.

10. Burstow DJ, Tajik AJ, Bailey KR, DeRemee RA, Taliercio CP (1989) Two dimensional echocardiographic findings in systemic sarcoidosis. Am J Cardiol 63: 478-82. doi: 10.1016/00029149(89)90323-8.

11. Mehta D, Lubitz SA, Frankel Z, Wisnivesky JP, Einstein AJ, Goldman M, Machac J, Teirstein A (2008)Cardiac involvement in patients with sarcoidosis: diagnostic and prognostic value of outpatient testing. Chest 133(6):1426-35. doi: 10.1378/chest.07-2784.

12. Freeman AM, Curran-Everett D, Weinberger HD, Fenster BE, Buckner JK, Gottschall EB, Sauer WH, Maier LA, Hamzeh NY (2013) Predictors of cardiac sarcoidosis using commonly available cardiac studies. Am J Cardiol 112: 280-5. doi: 10.1016/j.amjcard.2013.03.027.

13. Schouver ED, Moceri P, Doyen D, Tieulie N, Queyrel V, Baudouy D, Cerboni P, Gibelin P, Leroy S, Fuzibet JG, Ferrari E (2017) Early detection of cardiac involvement in sarcoidosis with 2-dimensional speckletracking echocardiography. Int J Cardiol 227:711-6. doi: 10.1016/j.ijcard.2016.10.073.

14. Chen J, Lei J, Scalzetti E, McGrath M, Feiglin D, Voelker R, Wang J, lannuzzi MC, Liu K (2018) Myocardial contractile patterns predict future cardiac events in sarcoidosis. Int J Cardiovasc Imaging 34(2):251-62. doi: 10.1007/s10554-017-1233-9.

15. Bayat F, Fahimi A, Tavana S, Tabary M, Khaheshi I (2020)Subclinical involvement of the heart and its associated factors in patients withsarcoidosiswith normal systolic function using 2D speckle tracking. Echocardiography 37(1):41-6. doi: 10.1111/echo.14572. 
16. Ise T, Hasegawa T, Morita Y, Yamada N, Funada A, Takahama H, Amaki M, Kanzaki H, Okamura H, Kamakura S, Shimizu W, Anzai T, Kitakaze M (2014) Extensive late gadolinium enhancement on cardiovascular magnetic resonance predicts adverse outcomes and lack of improvement in LV function after steroid therapy in cardiac sarcoidosis. Heart 100(15):1165-72. doi: 10.1136/heartjnl2013-305187.

17. Greulich S, Deluigi CC, Gloekler S, Wahl A, Zurn C, Kramer U, Nothnagel D, Bültel H, Schumm J, Grün S, Ong P, Wagner A, Schneider S, Nassenstein K, Gawaz M, Sechtem U, Bruder O, Mahrholdt H (2013) CMR imaging predicts death and other adverse events in suspected cardiac sarcoidosis. JACC Cardiovasc Imaging 6(4):501-11. doi: 10.1016/j.jcmg.2012.10.021.

18. Blankstein R, Kramer CM, Chandrashekhar Y (2017) The Challenges of Diagnosing Cardiac Sarcoidosis. JACC Cardiovasc Imaging 10(12):1534-6. doi: 10.1016/j.jcmg.2017.11.006.

19. Osborne MT, Hulten EA, Singh A, Waller AH, Bittencourt MS, Stewart GC, Hainer J, Murthy VL, Skali H, Dorbala S, Di Carli MF, Blankstein R (2014) Reduction in F-fluorodeoxyglucose uptake on serial cardiac positron emission tomography is associated with improved left ventricular ejection fraction in patients with cardiac sarcoidosis. J Nucl Cardiol 21(1):166-74. doi: 10.1007/s12350-013-9828-6.

20. Sperry BW, Tamarappoo BK, Oldan JD, Javed O, Culver DA, Brunken R, Cerqueira MD, Hachamovitch R (2018) Prognostic Impact of Extent, Severity, and Heterogeneity of Abnormalities on F-FDG PET Scans for Suspected Cardiac Sarcoidosis. JACC Cardiovasc Imaging 11(2 Pt 2):336-45. doi: 10.1016/j.jcmg.2017.04.020.

21. Blankstein R, Osborne M, Naya M, Waller A, Kim CK, Murthy VL, Kazemian P, Kwong RY, Tokuda M, Skali H, Padera R, Hainer J, Stevenson WG, Dorbala S, Di Carli MF (2014) Cardiac positron emission tomography enhances prognostic assessments of patients with suspected cardiac sarcoidosis. J Am Coll Cardiol 63(4):329-36. doi: 10.1016/j.jacc.2013.09.022.

22. Wang H, Song Y, Mu J, Shang J, Wang J, Ruan L (2020) Left ventricularsystolicdyssynchrony in patients with Kawasaki disease: a real-time three-dimensional echocardiography study. Int J Cardiovasc Imaging 36(10):1941-51. doi: 10.1007/s10554-020-01909-2.

23. Migrino RQ, Harmann L, Woods T, Bright M, Truran S, Hari P (2008) Intraventriculardyssynchronyin light chain amyloidosis: a new mechanism of systolic dysfunction assessed by 3-dimensional echocardiography. Cardiovasc Ultrasound 7;6:40. doi: 10.1186/1476-7120-6-40.

24. Guerra VC, Martins Lde M, Oliveira RM, da Silva KR, Binotto MA, Tsutsui JM, Kallil R, Costa R, Mathias W Jr (2015)Prevalence of Left VentricularDyssynchronyin Patients with Congenital Atrioventricular Block and Long-Term Pacing: A Three-Dimensional Echocardiographic Study. Echocardiography 32(9):1400-6. doi: 10.1111/echo.12871.

25. Tsuji T, Tanaka H, Matsumoto K, Miyoshi T, Hiraishi M, Kaneko A, Ryo K, Fukuda Y, Tatsumi K, Onishi T, Kawai H, Hirata KI (2013) Capability ofthree-dimensionalspeckle tracking radial strain for identification of patients with cardiac sarcoidosis. Int J Cardiovasc Imaging 29(2):317-24. doi: 10.1007/s10554-012-0104-7. 


\section{Figures}

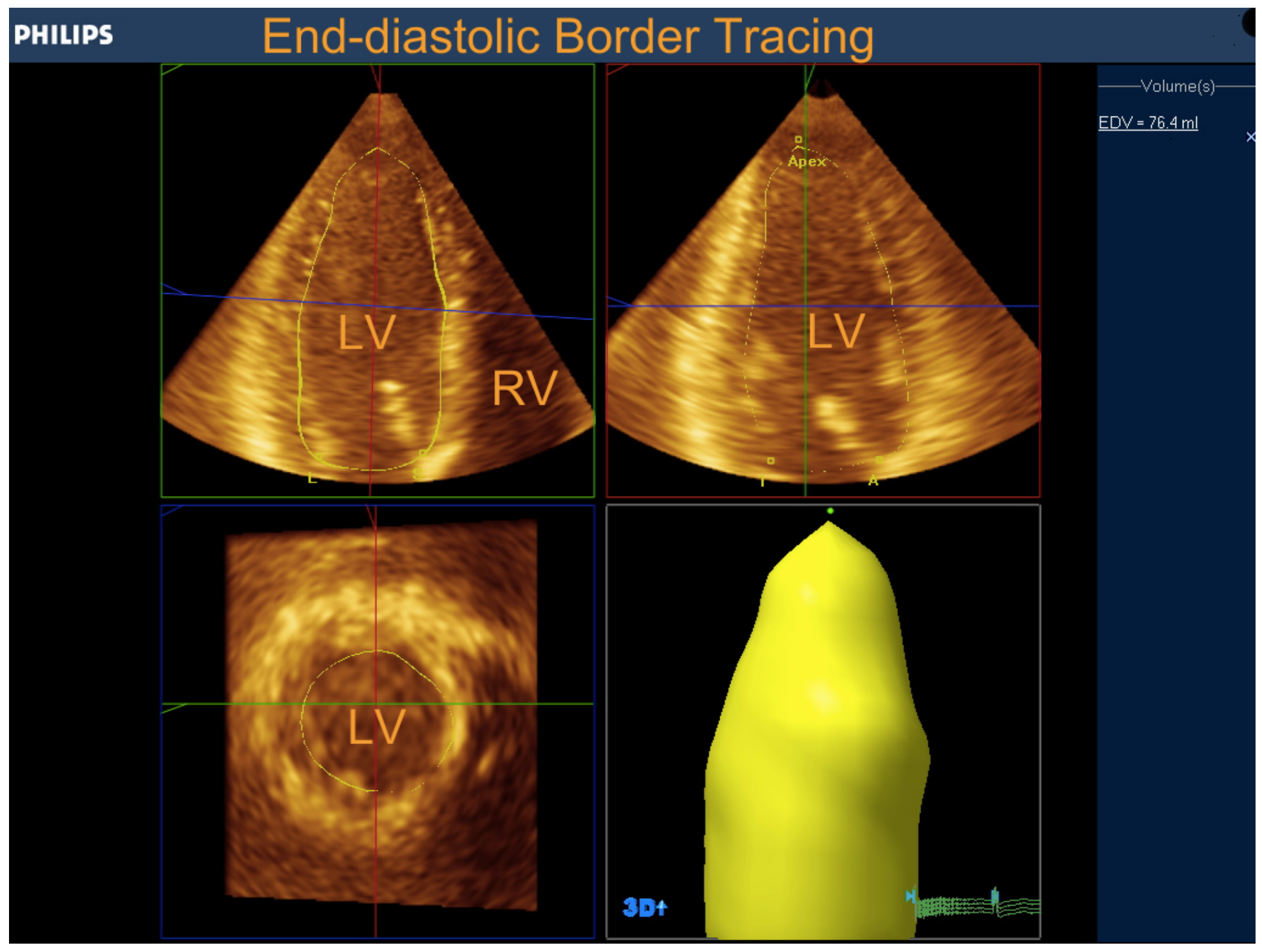

\section{Figure 1}

We adjusted transverse (at the level of papillary muscles) and saggital (from the midline of mitral annulus to apex) planes; then automatic border definition was performed by applying five points: anterior, inferior, septal, lateral annulus, and apex. Border definitions were manually modified in some of cases by including papillary muscles and trabeculations as parts of LV cavity 


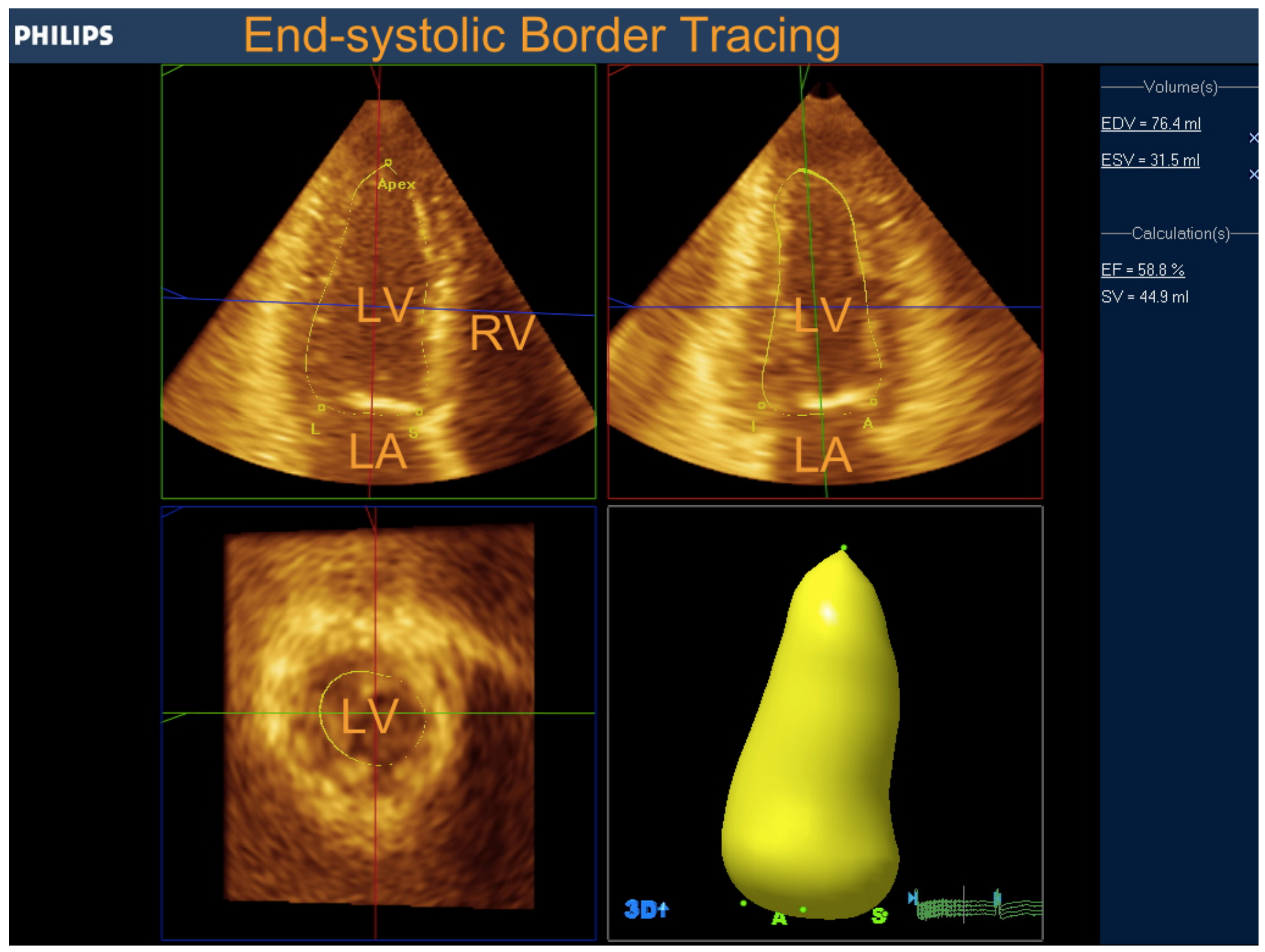

Figure 2

We performed sequence analysis at the end of the procedure and checked for border detection frame-byframe. 


\section{PHILIPS}

\begin{tabular}{|l|l|l|l|}
\hline Global & Regional (msec) & Regional (\%R-R) Parametric Imaging \\
\hline
\end{tabular}

\section{Regional (\%R-R) Report Page}

Volume(s)

$\underline{\underline{E D V}=76.4 \mathrm{ml}}$

$\mathrm{ESV}=31.5 \mathrm{ml}$

$\begin{array}{lrr}\text { Tmsv 16-SD* } & 5.32 & \% \\ \text { Tmsv 12-SD } & 2.13 & \% \\ \text { Tmsv 6-SD } & 1.06 & \% \\ \text { Tmsv 16-Dif } & 23.99 & \% \\ \text { Tmsv 12-Dif } & 7.62 & \% \\ \text { Tmsv 6-Dif } & 2.94 & \% \\ \text { Tmsv 3-6 } & 0.81 & \% \\ \text { Tmsv 3-5 } & 1.37 & \% \\ \text { Tmsv Sel-SD } & --- & \% \\ \text { Tmsv Sel-Dif } & --- & \% \\ \text { R-R Time } & 800 & \text { ms }\end{array}$

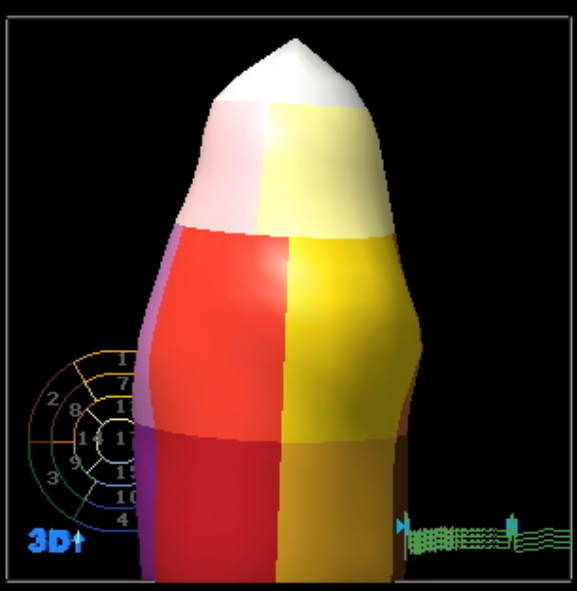

Calculation(s)

$\underline{E F}=58.8 \%$

$S \mathrm{~V}=44.9 \mathrm{ml}$

Regional

Tmsv Sel-SD = eroce

Tmsv Sel-Dif $=$ work

Tmsv Sel-SD =

*Excludes Segment 17

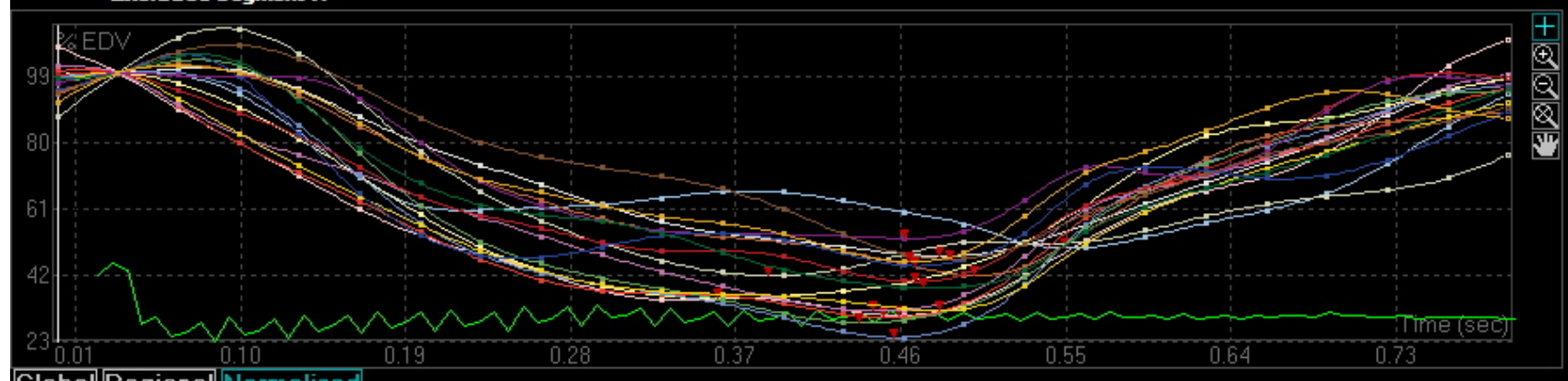

Global Reqional Normalized

\section{Figure 3}

LVEF, LVEDV, LVESV and 16 segments SDI (SDI_16) were obtained at the end of analysis and shown as a report page 